\title{
Conditioned Stimulus Familiarity Determines Effects of MK-801 on Fear Extinction
}

\author{
Wan Yee Macy Chan and Gavan P. McNally \\ University of New South Wales
}

\begin{abstract}
Six experiments studied the role of conditioned stimulus (CS) familiarity in determining the effects of the $N$-methyl-D-aspartate (NMDA) receptor antagonist MK-801 on fear extinction. Systemic administration of MK-801 $(0.1 \mathrm{mg} / \mathrm{kg})$ impaired initial extinction but not reextinction learning. MK-801 impaired reextinction learning when the CS was relatively novel during reextinction training but not initial extinction learning when the CS was relatively familiar during initial extinction training. A context change failed to reinstate the sensitivity of initial fear extinction learning about a relatively familiar CS to MK-801. These experiments show that CS familiarity is an important determinant of effects of MK-801 on fear extinction learning: MK-801 impaired extinction learning about novel stimuli but spared extinction learning about familiar stimuli.
\end{abstract}

Keywords: fear, extinction, NMDA, memory

Pavlovian conditioning has been extensively used to study mechanisms for the acquisition and loss of fear. Exposed to pairings of an initially innocuous stimulus (conditioned stimulus; CS), such as a light or a tone, with an innately aversive stimulus (unconditioned stimulus; US), such as a footshock, animals learn to fear the CS as indexed by expression of responses such as freezing, potentiated startle, and increased blood pressure upon later presentations of the CS. This learning is mediated, at least in part, by $N$-methyl-D-aspartate (NMDA) receptors. Systemic, intracerebroventricular or intraamygdala infusions of an NMDA receptor antagonist impair fear learning (Cox \& Westbrook, 1994; Kim, DeCola, Landeira-Fernandez, \& Fanselow, 1991; Miserendino, Sananes, Melia, \& Davis, 1990). The fear acquired via conditioning can be lost through extinction training. Like acquisition, fear extinction is dependent on NMDA receptors and their relevant signal transduction cascades in the basolateral amygdala (BLA; Myers \& Davis, 2007). Systemic and BLA microinjection of NMDA antagonist impairs fear extinction learning (Baker \& Azorlosa, 1996; Falls, Miserendino, \& Davis, 1992; Langton, Kim, Nicholas, \& Richardson, 2007; Sotres-Bayon, Bush, \& LeDoux, 2007). Conversely, both BLA and systemic administrations of the NMDA receptor partial agonist D-cycloserine (DCS) facilitate fear extinction learning (Ledgerwood, Richardson, \& Cranney, 2003; Walker, Ressler, Lu, \& Davis, 2002). Moreover, DCS facilitates loss of fear when combined with exposure-based therapy in human clinical populations (Guastella, Dadds, Lovibond, Mitchell, \&

Wan Yee Macy Chan and Gavan P. McNally, School of Psychology, University of New South Wales, Sydney, Australia.

This research was supported by Grant DP0877430 from the Australian Research Council to Gavan P. McNally. We thank Julia Langton, Vincent Laurent, Rick Richardson, and Fred Westbrook for their helpful discussions of these experiments.

Correspondence concerning this article should be addressed to Gavan P. McNally, School of Psychology, University of New South Wales, Sydney, 2052, Australia. E-mail: g.mcnally@unsw.edu.au
Richardson, 2007; Hofmann et al., 2006; Kushner et al., 2007; Ressler et al., 2004). Taken together, these findings have been interpreted to mean that NMDA receptors are obligatory for the acquisition and the loss of fear.

Recent findings, however, have challenged this view. For example, infusions of the NMDA receptor antagonist DL-APV into the BLA impaired initial fear extinction learning but not reextinction learning (Laurent, Marchand, \& Westbrook, 2008). In this experiment, rats in a "reextinction" condition were trained to fear a distinctive context. This fear was then extinguished. Rats were then retrained to fear the context prior to a second bout of extinction training. This second bout of extinction training, in contrast to the first, was unaffected by BLA infusions of DL-APV. Similar findings were recently reported for the NMDA receptor partial agonist DCS. Injection of DCS prior to initial extinction training of a discrete CS facilitated fear extinction learning, whereas such injections prior to a second bout of extinction training did not (Langton \& Richardson, in press). Finally, in some cases, such as the developing rat, initial fear extinction learning may be insensitive to NMDA receptor antagonism. For example, fear extinction learning in 16-day-old rats was not disrupted by MK-801 (Langton et al., 2007).

These findings suggest that the contributions of NMDA receptors to fear extinction learning are not invariant. Rather, other variables may also determine sensitivity of extinction learning to NMDA receptor antagonism. One such variable may be familiarity of the events being learned about. For example, an important difference between reextinction learning and initial extinction learning is that in the former the CS is more familiar than it is in the latter. It is well documented that familiarity of a CS is an important determinant of how an organism learns about it (Hall, 1991). Familiarity may likewise determine sensitivity of fear extinction learning to disruption by NMDA receptor antagonists. Indeed, studies of spatial learning have shown that pretraining on one maze can render later spatial learning in a second maze insensitive to disruption by NMDA receptor antagonism (Bannerman, Good, 
Butcher, Ramsay, \& Morris, 1995; Saucier \& Cain, 1995; Saucier, Hargreaves, Boon, Vanderwolf, \& Cain, 1996). Similar findings have been reported in other conditioning preparations, including contextual fear conditioning (Sanders \& Fanselow, 2003) and one-trial inhibitory avoidance learning (Roesler et al., 1998). Consistent with this interpretation, the transition from an NMDAdependent to NMDA-independent fear extinction process is stimulus specific. Reextinction learning was NMDA-independent only when the first and second extinction learning episodes involved the same CS (Langton \& Richardson, in press).

The experiments reported here studied the role of CS familiarity in determining the effects of an NMDA receptor antagonist on fear extinction. In particular, these experiments manipulated the familiarity of a CS during initial extinction learning or during reextinction learning to test two predictions. First, reextinction of a less familiar CS may be NMDA-dependent. Second, initial extinction of a familiar CS may be NMDA-independent. In other words, we examined the possibility that the involvement of NMDA receptors in fear extinction may be determined by CS familiarity regardless of whether a particular fear extinction episode represents an initial experience or a reexperience.

\section{Experiment 1}

The aim of Experiment 1 was to replicate previous findings that NMDA receptor antagonism prior to extinction training impairs long-term extinction in adult rats. We used the noncompetitive NMDA receptor antagonist MK-801 in this and remaining experiments because it has previously been shown to impair initial fear extinction learning (Baker \& Azorlosa, 1996; Cox \& Westbrook, 1994; Langton et al., 2007), but its effects on reextinction are unknown. It is worth emphasizing that the dissociation between extinction and reextinction has been observed with other NMDA receptor antagonists as well as with the partial agonist DCS (Langton \& Richardson, in press; Laurent et al., 2008). Consideration was given to using the NR2B selective antagonist ifenprodil, but the short half life of this drug upon systemic administration substantially reduces its usefulness for the experimental designs used here. The between-groups component of the experiment was a single-factor two-group design. Rats were trained to fear an auditory CS. This fear was then extinguished. Rats were injected with MK-801 or saline prior to extinction training. All rats were tested the next day for fear responses to the CS to assess long-term extinction retention.

\section{Method}

\section{Subjects}

The subjects were 16 experimentally naive, adult, male Wistar rats (265-353 g) obtained from a commercial supplier (Monash Animal Services, Gippsland, Victoria, Australia). Upon arrival, rats were housed in groups of 8 in plastic cages $(67 \mathrm{~cm}$ long $\times 30$ $\mathrm{cm}$ wide $\times 22 \mathrm{~cm}$ high) in a colony room maintained at $20-22^{\circ} \mathrm{C}$ on a 12-hr/12-hr light/dark cycle (lights on at 7 a.m.). Food and water were freely available. The rats were handled (1-2 min per rat per day) for 4 days prior to conditioning to habituate them to the experimenter. The procedures used in this and the following experiments were conducted in accordance with the Animal Re- search Act 1985 No. 123 and were approved by the Animal Care and Ethics Committee of the University of New South Wales.

\section{Behavioral Apparatus}

Conditioning and testing were conducted in a set of four identical chambers $(24 \mathrm{~cm}$ long $\times 30 \mathrm{~cm}$ wide $\times 21 \mathrm{~cm}$ high). The top and rear walls of these chambers as well as the front hinged door were constructed of clear Perspex, and the end walls were made of stainless steel. The floor in each chamber consisted of stainless steel rods $4 \mathrm{~mm}$ in diameter spaced $15 \mathrm{~mm}$ apart (center to center). Each chamber stood $2 \mathrm{~cm}$ above a tray of paper pellet bedding (Fibercycle, Mudgeeraba, Queensland, Australia). The chambers were cleaned with water, and the bedding underneath the chambers was changed between rats. These four chambers were located individually within sound-attenuating boxes that were painted white. The boxes were constantly illuminated by a single red LED such that levels of illumination within the conditioning chambers were 15 candela $/ \mathrm{m}^{2}$. Ventilation fans provided a constant background noise (67 $\mathrm{dB}$; A scale).

The CS was a $30-\mathrm{s} 750-\mathrm{Hz} 82-\mathrm{dB}$ (A scale) tone, delivered through a speaker mounted on the ceiling of each soundattenuating box. The footshock US was a 1-s, 0.8-mA unscrambled AC $50-\mathrm{Hz}$ shock from a constant current generator that was delivered to the floor of each chamber. The current available to each floor could be adjusted using an in-line milliampere meter. Digital videocameras were mounted on the rear wall of each box and connected to a digital multiplexer in an adjacent room that, in turn, was connected to a DVD recorder. The stimuli used for conditioning were controlled by computer (LabView, National Instruments, Austin, TX).

\section{Procedure}

Experiment 1 consisted of three stages: acquisition, extinction, and test.

Fear acquisition. On Day 1, rats were placed in the experimental chamber. After a 120-s adaptation period, the CS was presented for $30 \mathrm{~s}$. The shock US was administered during the last second of the CS presentation. Two more CS-US pairings were presented, with an intertrial interval (ITI) of $120 \mathrm{~s}$.

Extinction. On Day 2, rats were subcutaneously (s.c.) injected with MK-801 (0.1 mg/kg; Sigma-Aldrich, Sydney, New South Wales, Australia; $n=8)$ or saline $(n=8)$ in a volume of $1 \mathrm{ml} / \mathrm{kg}$. In this and the remaining experiments, group allocation was random. Ten minutes later, the rats were placed in the chambers for a $37-$ min session. There was a 60 -s adaptation period, followed by twelve 120-s presentations of the CS at an ITI of $60 \mathrm{~s}$.

Test. On Day 3, all animals were returned to the conditioning chambers and tested drug-free. The procedures on test were identical to those of extinction with the exception that the test involved three presentations of the CS. In this and the remaining experiments there were no injections prior to test.

\section{Statistics}

In this and the remaining experiments, performance during all stages was recorded. Rats were scored every $4 \mathrm{~s}$ as either freezing (defined as the absence of all movement other than that required 
for breathing) or not freezing. The number of observations scored as freezing during each observation period were summed and converted to a percentage. Test data were scored by two observers, one of whom was unaware of group allocations. In this and the remaining experiments the interrater reliability-that is, the correlation between the percentages of observations each rat was scored as freezing by each observer-exceeded 0.9 . The data were analyzed by means of a planned orthogonal contrast testing procedure, and the Type I error rate was controlled at 0.05 for each contrast tested.

\section{Results and Discussion}

The mean (plus or minus the standard error of the mean; SEM) percentage of observations scored as freezing across the three stages of the experiment are shown in Figure 1. Inspection of Figure 1 suggests that s.c. injection of MK-801 prior to extinction training impaired expression of freezing during extinction training and also impaired the development of long-term extinction as indexed by freezing to the CS on test. These observations were confirmed by the statistical analysis.

During acquisition, the average level of freezing across the three CS presentations was significantly higher than that of the pre-CS period, $F(1,15)=71.9, p<.05$. A within-subjects analysis showed that fear to the CS was acquired normally because freezing increased linearly across CS-US pairings, $F(1,15)=83.3, p<$ .05. During extinction, there was no significant difference in the level of pre-CS freezing between Group Saline and Group MK$801, F(1,14)=3.0, p>.05$. Averaged across drug conditions, there was a significant increase in freezing from pre-CS period to the first CS presentation, $F(1,14)=14.0, p<.05$. This interacted significantly with the drug factor, such that the increase in freezing was greater for Group Saline than for Group MK-801, $F(1,14)=$ $11.8, p<.05$. The average level of freezing across the 12 extinction CS presentations was higher for Group Saline than for Group MK-801, $F(1,14)=17.9, p<.05$. This shows that injection of MK-801 prior to extinction training disrupted expression of freezing. Averaged across groups, there was evidence for withinsession extinction because freezing decreased in a significant linear fashion across CS presentations, $F(1,14)=13.2, p<.05$. This within-session extinction interacted significantly with the drug factor, $F(1,14)=10.9, p<.05$.

The data of primary interest are those from test and are shown in the right-hand panel of Figure 1. On test, there was no signif-
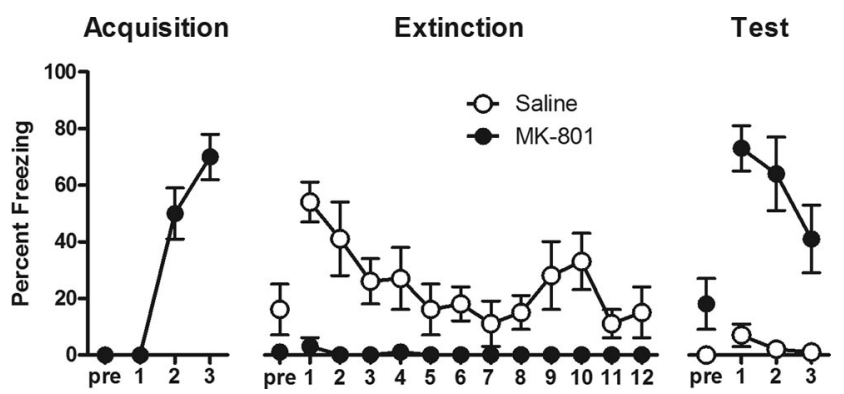

Figure 1. Mean and SEM levels of freezing during the three stages of Experiment 1. Systemic MK-801 $(0.1 \mathrm{mg} / \mathrm{kg})$ administered prior to extinction training impaired long-term extinction learning. icant difference in pre-CS levels of freezing between Group MK801 and Group Saline, $F(1,14)=4.4, p>.05$. There was overall significantly more freezing to the CS than during the pre-CS period, $F(1,14)=19.2, p<.05$, and this difference interacted significantly with the difference between groups, $F(1,14)=14.3$, $p<.05$. Importantly, Group MK-801 exhibited significantly more freezing than did Group Saline during test presentations of the CS, $F(1,14)=36.1, p<.05$. This shows that MK-801 impaired extinction learning. There was a significant reduction in freezing across test trials, $F(1,14)=18.5, p<.05$, and this was greater in Group MK-801 than in Group Saline, $F(1,14)=8.9, p<.05$.

This experiment has confirmed that initial fear extinction is mediated by NMDA receptors (Baker \& Azorlosa, 1996; Langton et al., 2007). Specifically, injection of the noncompetitive NMDA receptor antagonist MK-801 prior to extinction training impaired extinction learning, as evidenced by increased fear to the auditory $\mathrm{CS}$ on a drug-free test, in comparison with a group injected with saline prior to extinction training. Injection of MK-801 prior to extinction training also significantly disrupted expression of the freezing response during extinction training. This disruption of freezing was most likely due to motoric effects of the drug. It is possible that the disruption of extinction learning was caused by this disruption of freezing during extinction training. This possibility is unlikely in light of the results of the remaining experiments but is considered in the General Discussion.

\section{Experiment 2}

Experiment 1 confirms that systemic injection of MK-801 impaired extinction learning. Experiment 2 examines whether the NMDA receptor antagonist MK-801 likewise impairs fear reextinction learning. It has previously been demonstrated that infusions of the competitive NMDA receptor antagonist APV into BLA selectively disrupted fear extinction but not reextinction learning (Laurent et al., 2008). Furthermore, Langton and Richardson (in press) found that systemic administration of the NMDA receptor partial agonist DCS facilitated initial extinction but not reextinction learning. These findings suggest that reextinction learning, unlike initial extinction learning, can be insensitive to NMDA receptor antagonism or partial agonism. Experiment 2 examines the role of NMDA receptors in fear reextinction by assessing the effects of systemically administered MK-801 on reextinction learning. The between-groups component of the experiment was a single-factor two-group design. Both groups received initial training to fear an auditory CS, followed by initial fear extinction. This was followed by retraining to fear the auditory CS and then reextinction. Rats were injected with MK-801 or saline prior to reextinction training. All rats were tested the next day for fear responses to the CS to assess long-term reextinction retention.

\section{Method}

\section{Subjects and Apparatus}

The subjects were 16 experimentally naive, adult, male Wistar rats (232-292 g) obtained from the same source and maintained under the same conditions as in Experiment 1. The apparatus was identical to that used in Experiment 1. 


\section{Procedure}

Experiment 2 consisted of five stages: acquisition, extinction, reacquisition, reextinction, and test. One stage took place each day across 5 consecutive days. The procedure for acquisition was identical to that in Experiment 1. The procedure for extinction was similar to that in Experiment 1 with the single exception that no drugs were administered. The procedure for reacquisition was identical to that for acquisition. The procedure for reextinction was similar to that for extinction except that rats were injected s.c. with either MK-801 $(0.1 \mathrm{mg} / \mathrm{kg} ; n=8)$ or saline $(n=8) 10 \mathrm{~min}$ prior to reextinction. The procedure for test was identical to that in Experiment 1 .

\section{Results and Discussion}

The mean $( \pm$ SEM) percentages of observations scored as freezing across the five stages of Experiment 2 are shown in Figure 2. Inspection of Figure 2 suggests that fear was acquired, extinguished, and reacquired normally. Importantly, Figure 2 suggests that s.c. injection of MK-801 prior to reextinction training impaired expression of freezing during reextinction training but did not impair the development of long-term reextinction. These observations were confirmed by the statistical analysis.

During fear acquisition the average level of freezing across the three CS presentations was significantly higher than that of the pre-CS period, $F(1,15)=25.4, p<.05$. A within-subject analysis showed that fear was acquired normally because there was a significant linear increase in freezing across CS-US pairings, $F(1$, $15)=36.1, p<.05$.

During extinction, there was a significant increase in freezing from the pre-CS period to the first CS presentation, $F(1,15)=$ $18.5, p<.05$. Moreover, there was a significant linear decrease in freezing across the extinction session, $F(1,15)=14.4, p<.05$. These results confirm that the extinction training caused significant within-session extinction.

During reacquisition, the average level of freezing across the three CS presentations was significantly higher than that of the pre-CS period, $F(1,15)=80.6, p<.05$. A within-subject analysis showed that fear was reacquired normally because there was a significant linear increase in freezing across CS-US pairings, $F(1$, $15)=16.7, p<.05$.

The data of primary interest are those from reextinction and test. During reextinction, there was a significant difference in the level of pre-CS freezing between Group Saline and Group MK-801, $F(1,14)=70.3, p<.05$. Averaged across drug conditions, there was a significant increase in freezing from the pre-CS period to the first CS presentation, $F(1,14)=7.3, p<.05$. This increase in freezing from pre-CS to the first CS significantly interacted with the drug factor, such that this increase in freezing was greater for Group Saline than for Group MK-801, $F(1,14)=7.3, p<.05$. The average level of freezing across the 12 nonreinforced CS presentations was higher for Group Saline than for Group MK$801, F(1,14)=45.8, p<.05$, indicating disruption of freezing by MK-801. Averaged across groups, there was evidence for withinsession extinction because there was a significant linear decrease in freezing across the reextinction session, $F(1,14)=64.3, p<$ .05 . This decrease in freezing interacted significantly with the drug factor, $F(1,14)=64.3, p<.05$. Taken together, these results confirm that the reextinction training caused significant withinsession extinction and that MK-801 administration disrupted expression of the freezing response.

On test, there was no significant difference in pre-CS levels of freezing between rats treated with MK-801 and those treated with saline prior to reextinction training, $F(1,14)<1, p>.05$. There was overall significantly more freezing during the CS than during the pre-CS period, $F(1,14)=15.9, p<.05$, and this did not differ between groups, $F(1,14)=2.5, p>.05$. Importantly, there was no overall significant difference between Group MK-801 and Group Saline in freezing across test presentations of the CS, $F(1$, 14) $\langle 1, p>.05$. There was no overall significant reduction in freezing across test trials, $F(1,14)=3.1, p>.05$, and no Group $\times$ Trial interaction, $F(1,14)<1, p>.05$.

Experiment 1 showed that injection of MK-801 prior to extinction training impaired the expression of the freezing $\mathrm{CR}$ and also the development of long-term extinction. The results of Experiment 2 show that although injection of MK-801 disrupted the expression of the freezing CR during reextinction training, such injections did not impair long-term reextinction as indexed by the absence of differences between groups on test. Therefore, Experiment 2 extends previous demonstrations through the use of a discrete auditory CS and confirms that the initial extinction of conditioned fear and the reextinction of conditioned fear can be differentially affected by NMDA receptor antagonism. Initial extinction was disrupted by MK-801, whereas reextinction was not. The absence of an effect of MK-801 on reextinction also suggests that the impairment of initial extinction by MK-801 in Experiment 1 cannot be attributed to the tendency of the drug to disrupt fear CR expression. In both Experiments 1 and 2, injection of MK-801 disrupted expression of freezing during extinction (Experiment 1) or reextinction (Experiment 2) training. However, injection of

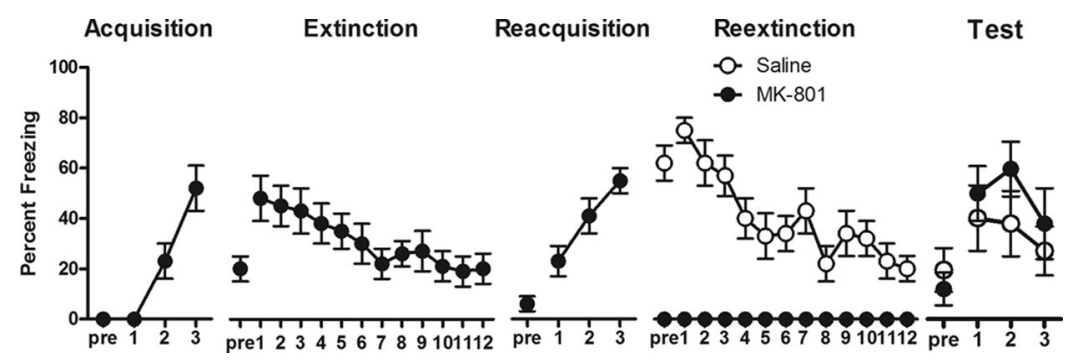

Figure 2. Mean and SEM levels of freezing during the five stages of Experiment 2. Systemic MK-801 (0.1 $\mathrm{mg} / \mathrm{kg}$ ) administered prior to reextinction training did not impair long-term reextinction learning. 
MK-801 only disrupted extinction learning (Experiment 1). It had no significant effect on reextinction learning (Experiment 2).

\section{Experiment 3}

Experiments 1 and 2 add to the current literature suggesting that learning and relearning of extinction may involve different processes. In particular, MK-801 impaired extinction learning but not reextinction learning. It has been suggested that, unlike initial extinction learning, reextinction may trigger retrieval of the original extinction memory, and this may not require further plasticity or NMDA receptor function. Such an explanation of the dissociation between extinction and reextinction is plausible and consistent with the existing empirical data. However, other interpretations are possible. One potentially important difference between extinction and reextinction is CS familiarity. By definition, the CS is more familiar at reextinction training than at initial extinction training. Experiments examining differences in the mechanisms for initial extinction learning and reextinction learning potentially confound differences in CS familiarity during each of these processes. If CS familiarity is a determinant of NMDA receptor contributions to fear extinction, one prediction that follows is that reextinction learning involving a less familiar (relatively novel) CS should remain sensitive to NMDA receptor antagonism. Experiment 3 tests this prediction using a two-group (saline vs. MK-801) reextinction design. The procedure was similar to Experiment 2 with the difference being that only half the number of CS presentations was used for extinction and reextinction. This was intended to reduce the familiarity, or maintain the novelty, of the CS.

\section{Method}

The subjects were 15 experimentally naive, adult, male Wistar rats $(272-371 \mathrm{~g})$ obtained from the same source and maintained under the same conditions as in Experiment 1. There were 8 subjects in Group Saline and 7 subjects in Group MK-801. The apparatus was identical to that used in Experiment 1.

The procedures used in acquisition, extinction, reacquisition, reextinction, and test were identical to those in Experiment 2, with the exception that extinction and reextinction involved 6 nonreinforced CS presentations (instead of 12 CS-only presentations as in Experiment 2). This meant that session lengths for extinction and reextinction were $18 \mathrm{~min}$.

\section{Results and Discussion}

The mean $( \pm$ SEM) percentage of observations scored as freezing across the five stages is shown in Figure 3. Inspection of Figure 3 suggests that s.c. injection of MK-801 prior to reextinction training impaired both the expression of freezing during reextinction training and the development of long-term reextinction. These observations were confirmed by the statistical analysis.

During acquisition, the average level of freezing across the three CS presentations was significantly higher than that of the pre-CS period, $F(1,14)=188.0, p<.05$. Fear was acquired normally because within-subject analysis showed a significant linear increase in freezing across CS-US pairings, $F(1,14)=271.6, p<$ .05. During extinction, there was a significant increase in freezing from the pre-CS period to the first CS presentation, $F(1,14)=$ $66.5, p<.05$. Moreover, there was a significant linear decrease in freezing across the extinction session, $F(1,14)=56.2, p<.05$. This confirms that extinction training caused significant withinsession extinction.

During reacquisition, the average level of freezing across the three CS presentations was significantly higher than that of the pre-CS period, $F(1,14)=155.6, p<.05$. A within-subject analysis showed that fear was acquired normally across CS-US pairings because there was a significant linear increase in freezing across fear reacquisition training, $F(1,14)=17.0, p<.05$.

During reextinction, there was no significant difference in the level of pre-CS freezing between Group Saline and Group MK$801, F(1,13)=4.2, p>.05$. Averaged across drug conditions, there was no significant increase in freezing from the pre-CS period to the first CS presentation, $F(1,13)=4.1, p>.05$. However, the increase in freezing from pre-CS to the first CS presentation interacted significantly with the drug factor, such that this increase in freezing was greater for Group Saline than Group MK-801, $F(1,13)=12.0, p<.05$. The average level of freezing across the six nonreinforced CS presentations was higher for Group Saline than for Group MK-801, $F(1,13)=72.9, p<.05$, indicating disruption of freezing by MK-801. There was evidence for reextinction because averaged across groups there was a significant linear decrease in freezing across the reextinction session, $F(1,13)=7.7, p<.05$. This decrease in freezing did not interact significantly with the difference between groups, $F(1,13)=2.7$, $p>.05$.

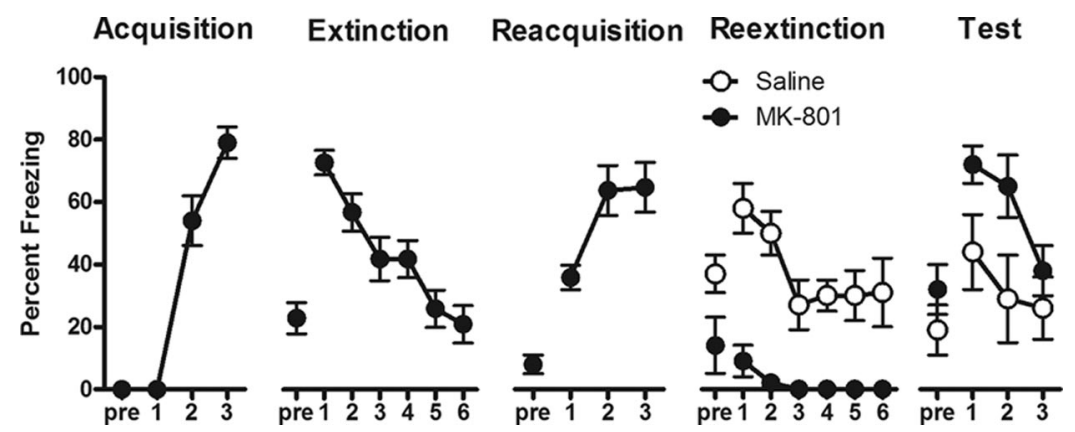

Figure 3. Mean and SEM levels of freezing during the five stages of Experiment 3. Systemic MK-801 (0.1 $\mathrm{mg} / \mathrm{kg}$ ) administered prior to reextinction training of a relatively novel conditioned stimulus impaired long-term reextinction learning. 
On test, there was no significant difference in pre-CS levels of freezing between Group MK-801 and Group Saline, $F(1,13)=$ $1.3, p>.05$. There was significantly more overall freezing to the $\mathrm{CS}$ than during the pre-CS period, $F(1,13)=12.2, p<.05$, and this did not differ between groups, $F(1,13)=2.5, p>.05$. Importantly, Group MK-801 exhibited a significantly higher level of freezing in comparison with Group Saline across Test CS presentations, $F(1,13)=5.6, p<.05$. There was no overall significant reduction in freezing across test trials, $F(1,13)=3.1$, $p>.05$, and no Group $\times$ Trial interaction, $F(1,13)<1, p>.05$.

Experiment 3 tested the possibility that familiarity of the auditory CS is an important determinant of the effects of MK-801 on reextinction learning. The design was similar to that used in Experiment 2. The difference between Experiment 3 and Experiment 2 was procedural. In Experiment 3, the procedure for extinction and reextinction involved half the number of CS presentations in comparison with that in Experiment 2. This modification was designed to reduce the familiarity, or maintain the novelty, of the $\mathrm{CS}$ during reextinction. The results using this modified procedure show that, in contrast to Experiment 2, injection of MK-801 prior to reextinction training significantly impaired fear reextinction learning. Again, injection of MK-801 prior to reextinction training disrupted expression of the freezing response. However, again, this disruption appeared unrelated to the results on test (see the General Discussion).

\section{Experiment 4}

The results of Experiment 3 support the possibility that CS familiarity is a determinant of the effects of MK-801 on fear reextinction. The aim of Experiment 4 was to test a second prediction that can be derived from the suggestion that CS familiarity is a determinant of the sensitivity of an extinction learning episode to NMDA receptor antagonism. Just as Experiment 3 demonstrated that reextinction involving a relatively novel CS was NMDA-dependent, initial extinction involving a relatively familiar CS should be NMDA-independent and thus unaffected by NMDA receptor antagonism. In Experiment 4 we used a preexposure manipulation to familiarize subjects with the CS prior to conditioning and extinction (Hall, 1991). The between-groups component of the experiment was a single-factor two-group design. Both groups received nonreinforced preexposures to the auditory CS followed by fear conditioning and then fear extinction. Rats were injected with MK-801 or saline prior to extinction training. All rats were tested the next day for fear responses to the CS to assess long-term extinction retention.

\section{Method}

The subjects were 16 experimentally naive, adult, male Wistar rats $(308-383 \mathrm{~g})$ obtained from the same source and maintained under the same conditions as in Experiment 1 . There were 8 subjects in Group Saline and 8 subjects in Group MK-801. The apparatus was identical to that used in Experiment 1.

There were four stages: preexposure, acquisition, extinction, and test, which took place one stage per day across 4 consecutive days. Preexposure occurred on Day 1 and involved 12 CS-only presentations. The procedure for these preexposures was identical to that used in extinction training in Experiment 1. Procedures for acquisition, extinction, and test were otherwise identical to those used in Experiment 1 .

\section{Results and Discussion}

The mean $( \pm$ SEM $)$ percentage of observations scored as freezing across the four stages is shown in Figure 4. Inspection of Figure 4 suggests that s.c. injection of MK- 801 prior to extinction training impaired expression of freezing during extinction training but not the development of long-term extinction as indexed by comparable levels of freezing to the CS on test between Group MK-801 and Group Saline. These observations were confirmed by the statistical analysis.

There was no freezing observed during the $12 \mathrm{CS}$ presentations of preexposure. During conditioning, the average level of freezing across the three CS presentations was significantly higher than that of the pre-CS period, $F(1,15)=44.0, p<.05$. A within-subject analysis showed a significant linear increase in freezing during fear acquisition training, $F(1,15)=55.7, p<.05$. During extinction, there were significantly higher levels of pre-CS freezing in Group Saline than in Group MK-801, $F(1,14)=6.3, p<.05$. Averaged across groups, there was a significant increase in freezing from pre-CS to first CS presentation, $F(1,14)=11.8, p<.05$. This increase in freezing interacted significantly with the drug factor, such that it was greater for Group Saline than for Group MK-801, $F(1,14)=11.8, p<.05$. The average level of freezing across the extinction CS presentations was higher for Group Saline

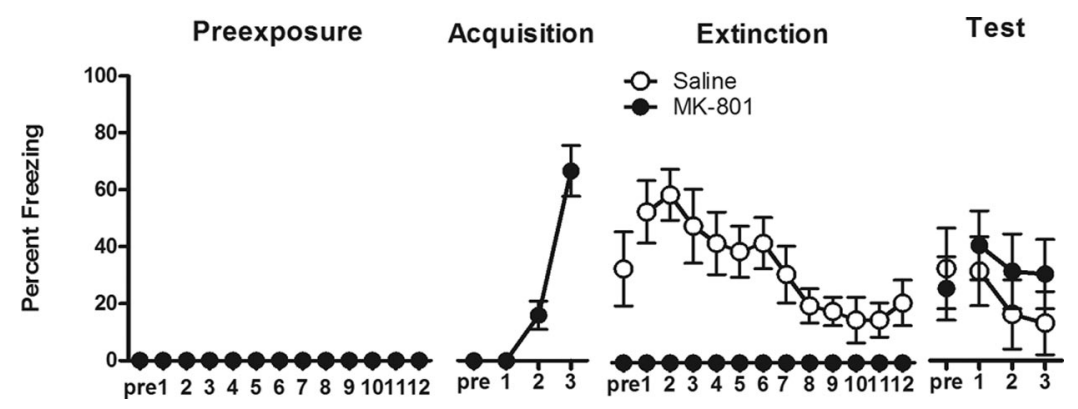

Figure 4. Mean and SEM levels of freezing during the four stages of Experiment 4. Systemic MK-801 (0.1 $\mathrm{mg} / \mathrm{kg}$ ) administered prior to extinction training of a relatively familiar conditioned stimulus did not impair long-term extinction learning. 
than for Group MK-801, $F(1,14)=26.3, p<.05$, indicating disruption of freezing by injections of MK-801. Moreover, averaged across groups, there was a significant linear decrease in freezing across the extinction session, $F(1,14)=22.5, p<.05$. This decrease in freezing interacted significantly with the drug factor, $F(1,14)=22.5, p<.05$. These results confirm that extinction training caused significant within-session extinction and that MK-801 administration led to disruption of the freezing response.

On test, there was no significant difference between Group MK-801 and Group Saline in the level of pre-CS freezing, $F(1$, $14)\langle 1, p>.05$. There was no overall significant difference in freezing to the CS in comparison with that of the pre-CS period $F(1,14)<1, p>.05$, and this did not differ between groups, $F(1$, $14)=3.7, p>.05$. There was no overall significant difference between Group MK-801 and Group Saline in freezing across test presentations of the CS, $F(1,14)<1, p>.05$. There was a significant reduction in freezing across test trials, $F(1,14)=12.0$, $p<.05$, but no Group $\times$ Trial interaction, $F(1,14)=1.3, p>.05$.

These results show that injection of MK-801 prior to initial extinction training of a familiar CS did not impair initial extinction learning. These results stand in contrast to the impairment of extinction learning produced by MK-801 in Experiment 1 when the CS had not been preexposed.

\section{Experiment 5}

The results of the previous experiments are consistent with the claim that CS familiarity is a critical determinant of the effects of NMDA receptor antagonism on fear extinction. The results of Experiment 4 are important in this regard because they show that initial extinction learning can proceed independently of NMDA receptor antagonism. However, the conclusion that CS familiarity determines the effects of NMDA receptor antagonism on fear extinction learning relies on between-experiments comparisons. For example, a comparison between Experiment 1 and Experiment 4 is required for the inference that MK-801 impaired extinction learning about a relatively novel but not relatively familiar CS. The first aim of the Experiment 5 was to overcome this limitation by providing a direct, within-experiment comparison of the effects of MK-801 on extinction of a relatively novel CS versus a relatively familiar CS. The second aim was to test a possible mechanism of CS familiarity. One such mechanism supposes that subjects learn about the relationship between the CS and the physical context in which it is presented (Wagner, 1981). Thus, a CS is familiar or unsurprising by virtue of being consistently presented in the same context. Presentation of that CS in a second, different context should reinstate the novelty of the CS.

The between-groups component of the experiment involved a $3 \times 2$ factorial design. The first factor was type and location of CS preexposure (None, Same, or Different). Groups None received no preexposure to either the contexts or the CS but received the same handling and transportation as did the remaining groups. Groups Same received preexposures to the CS in the same context as conditioning, extinction, and test, whereas Groups Different received such preexposures in a different context. The second factor was type of s.c. injection prior to initial extinction training (Saline or MK-801). Extinction learning should be impaired in Group None-MK-801 because the CS is relatively novel during extinc- tion training. Extinction learning should proceed normally in Group Same-MK-801 because the CS is relatively familiar during extinction training. The question of interest here concerned extinction learning in Group Different-MK-801. Specifically, if preexposures enable animals to form context-CS associations and such associations influence CS familiarity or sensitivity to MK-801 or both, then presentation of that CS in a second, different context during conditioning and extinction should reinstate sensitivity of CS extinction learning to MK-801.

\section{Method}

The subjects were 48 experimentally naive, adult, male Wistar rats $(260-505 \mathrm{~g})$ obtained from the same source and maintained under the same conditions as in Experiment 1. The apparatus was identical to that used in Experiment 1, with the only exception that CS preexposure for Groups Different took place in a different set of four chambers $(20.4 \mathrm{~cm}$ long $\times 23.4 \mathrm{~cm}$ wide $\times 19.5 \mathrm{~cm}$ high $)$. The front and rear walls as well as the hinged lid of these chambers were made of clear Perspex, and the side walls were made of stainless steel. The floor consisted of cardboard. Each chamber stood $3.5 \mathrm{~cm}$ above a tray of paper pellet bedding (Fibercycle, Mudgeeraba, Queensland, Australia) into which was placed $1 \mathrm{ml}$ of dilute rose oil. The chambers were cleaned with a damp paper towel, and bedding was changed between rats. These chambers were located individually within sound-attenuating boxes, the inner walls of which were painted black. The boxes were illuminated with a white 24-v light. An extractor fan in the rear wall of each box was operating during all sessions. Thus, the two sets of chambers differed in their visual, tactile, and olfactory properties. Experiment 5 consisted of four stages: preexposure, acquisition, extinction, and test, which took place one stage per day across 4 consecutive days. For Groups Same (Same-Saline, $n=8$; SameMK-801, $n=8$ ) and Groups Different (Different-Saline, $n=8$; Different-MK-801, $n=8$ ), preexposure on Day 1 was identical to procedures used in Experiment 4 and involved 12 CS-only presentations in either the same context or different context used for the remaining stages. For Group None (None-Saline, $n=8$; None-MK-801, $n=8$ ), animals were transported to the laboratory, briefly handled and left outside the laboratory for the same amount of time as were Groups Preexposed. Procedures were otherwise identical to those used in Experiment 1.

\section{Results and Discussion}

The mean $( \pm$ SEM $)$ percentage of observations scored as freezing across the four stages is shown in Figure 5. Inspection of Figure 5 suggests that s.c. injection of MK-801 prior to extinction training impaired expression of freezing during extinction training. Preextinction MK-801 administration impaired the development of long-term extinction for non-preexposed animals, such that Group None-MK-801 displayed more freezing than did Group NoneSaline. In contrast, MK-801 did not appear to impair extinction among Group Different and Group Same, as was indexed by comparable levels of freezing to the CS on test regardless of drug condition. Furthermore, the hypothesized effect of context change was not apparent. These observations were confirmed by the statistical analysis. 


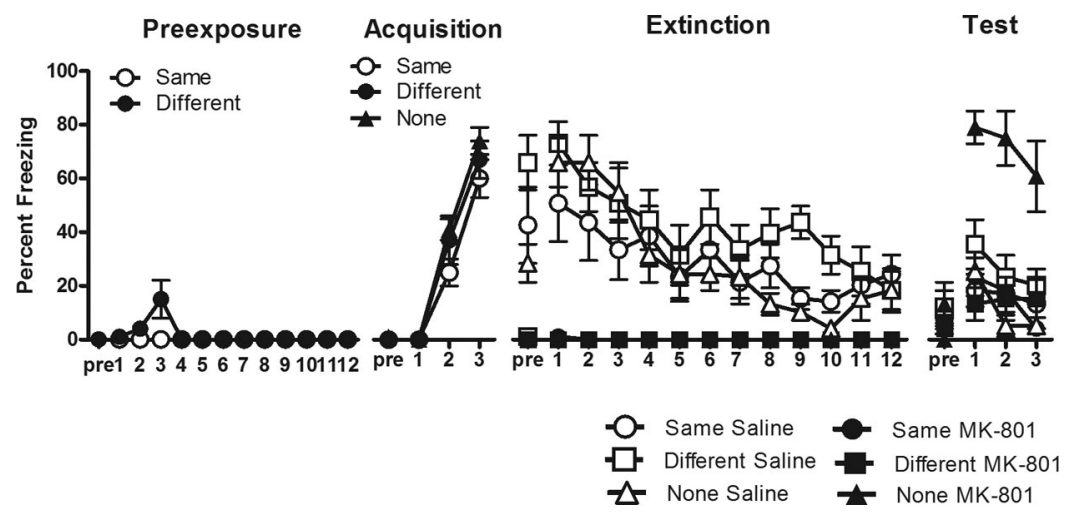

Figure 5. Mean and SEM levels of freezing during Experiment 5. Systemic MK-801 (0.1 mg/kg) administered prior to initial extinction training impaired long-term extinction learning of a relatively novel conditioned stimulus (CS; Groups None) but not of a relatively familiar CS (Groups Same and Different).

There were minimal levels of freezing during preexposure to the 12 CS presentations for the preexposed groups. During conditioning, the average level of freezing across the three CS presentations was significantly higher than that of the pre-CS period, averaged across all preexposure conditions, $F(1,45)=259.4, p<.05$. A within-subject analysis showed a significant linear increase in freezing during fear acquisition training, $F(1,45)=343.6, p<$ .05 . There were neither significant differences between groups during acquisition nor significant Group $\times$ Linear interactions, $F_{\mathrm{s}}(1,45)<2.5, p>.05$. This shows that all groups acquired fear of the CS at similar rates.

During extinction, there was a significantly higher level of pre-CS freezing in the Saline groups than in the MK-801 groups, averaged across preexposure conditions, $F(1,42)=53.0, p<.05$. Averaged across drug conditions, there was no significant effect of preexposure manipulation on pre-CS freezing, $F(1,42)=3.8, p>$ .05 , nor was there an effect of context (Same vs. Different), $F(1$, $42)=2.3, p>.05$. There were significant interactions between pre-CS versus first-CS freezing and Group Saline versus Group MK-801, $F(1,42)=21.4, p<.05$ (showing that MK-801 disrupted the increase in freezing), and Group None versus Group Preexposed, $F(1,42)=13.1, p<.05$ (showing that the increase in freezing was greater for non-pre-exposed animals), and there was also a three-way Drug $\times$ Preexposure $\times$ Pre-CS versus First-CS Freezing interaction, $F(1,42)=13.4, p<.05$ (showing that the effect of MK-801 on this increase in freezing was greater for non-pre-exposed groups).

The average level of freezing across the extinction CS presentations was higher for Group Saline than for Group MK-801, F(1, $42)=80.8, p<.05$, indicating disruption of freezing by injections of MK-801. There was no significant effect of preexposure (None vs. Preexposed) or context (Same vs. Different), $F \mathrm{~s}(1,42)<1.8$, $p>.05$. There was an overall significant linear decrease in freezing across the extinction session, $F(1,42)=50.1, p<.05$. This decrease in freezing interacted significantly with the drug factor, such that within-session extinction was greater for Group Saline than for Group MK-801, $F(1,42)=49.3, p<.05$, but did not interact significantly with the preexposure factor (None vs. Preexposed; Same vs. Different), $F_{\mathrm{s}}(1,42)<3.3, p>0.05$. These results confirm that extinction training caused significant within- session extinction and that MK-801 administration led to disruption of the freezing response.

On test, there were no significant group differences or interactions in levels of pre-CS freezing, $F_{\mathrm{S}}(1,42)<2.4, p>.05$. There was significantly more freezing during the first CS presentation than during the pre-CS period, $F(1,42)=46.3, p<.05$, averaged across groups. This difference interacted significantly with the difference between Group None and Group Preexposed, $F(1$, $42)=18.8, p<.05$, and Group MK-801 and Group Saline, $F(1$, $42)=12.0, p<.05$, as well as a three-way interaction with Group None versus Group Preexposed and Group MK-801 versus Group Saline, $F(1,42)=12.9, p<.05$.

During test CS presentations, there was a significant main effect of drug (MK-801 vs. Saline), $F(1,42)=21.4, p<.05$. There was also a significant main effect of preexposure (None vs. Preexposed), $F(1,42)=19.6, p<.05$, but no main effect of context (Same vs. Different), $F(1,42)=1.0, p>.05$. Importantly, there was a significant Drug $\times$ Preexposure interaction, such that the extinction impairment produced by MK-801 was greater among non-pre-exposed groups than among preexposed groups, $F(1$, $42)=25.9, p<.05$. These results confirm that MK-801 impaired extinction learning about a relatively novel CS but not a relatively familiar CS. There was, however, no significant Drug $\times$ Context of Preexposure interaction, $F(1,42)<1, p>.05$. This shows that the context change between preexposure and the remaining stages of the experiment did not reinstate sensitivity of extinction of a familiar CS to the effects of MK-801. There was a significant reduction in freezing across test trials, $F(1,42)=14.4, p<.05$, but there were no interactions with group, $\left.F_{\mathrm{S}}(1,42)<3.9, p\right\rangle$ .05 .

These results confirm that MK-801 impaired extinction learning about a relatively novel CS but not about a relatively familiar CS and that the context change between preexposure and the remaining stages of the experiment did not reinstate sensitivity of extinction of a familiar CS to the effects of MK-801. The finding that MK-801 impaired initial fear extinction learning about a relatively novel but not familiar CS provides a within-experiment replication of the results reported in Experiments 1 and 4. It is worth noting that the amount of extinction observed in Group None-Saline was slightly less in Experiment 5 than for the saline control group in 
Experiment 1. That is, comparison of Figure 1 with the remaining figures suggests that extinction was greater in Experiment 1 than in other experiments. In Experiment 1, the saline control displayed $7 \%$ freezing on test during CS1 in comparison with $37 \%$ (Experiment 2), 44\% (Experiment 3), 31\% (Experiment 4), and 25\% (Experiment 5 Group None-Saline) in each of the remaining experiments. This difference is of little concern because the impairment of initial extinction learning by MK-801 was observed in both Experiments 1 and 5. This shows that the impairment of initial extinction learning by MK-801 in these experiments was not simply due to differences in level of freezing among the relevant control groups across experiments. Likewise, the withinexperiment demonstration that MK-801 impaired initial fear extinction learning about a relatively novel CS but not a relatively familiar CS argues strongly against the possibility that the impact of CS familiarity is due to differences in levels of freezing among the control groups. Rather, Experiment 5 confirms that CS familiarity is an important determinant of the effects of MK-801 on fear-extinction learning.

\section{Experiment 6}

The design used in each of the experiments reported here confounds the potentially separable influences of familiarity on learning about the target CS versus the conditioning context. For example, the No Preexposure control condition used in Experiment 5 equated groups on handling and transport but not on exposure to the same and different contexts. Therefore it remains possible that the critical component of preexposure that determines the effectiveness of MK-801 on CS fear extinction is not familiarity of the CS but rather familiarity of the conditioning context. Our final experiment tested this possibility. The between-groups component involved two groups: Group Saline and Group MK-801 injected with saline or MK-801 prior to extinction training. Both groups received context preexposure, then conditioning of an auditory CS prior to extinction and test of fear to that CS. The question of interest was whether injection of MK-801 would impair extinction learning.

\section{Method}

The subjects were 16 experimentally naive, adult, male Wistar rats $(360-450 \mathrm{~g})$ obtained from the same source and maintained under the same conditions as in Experiment 1. There were 8 rats in Group MK-801 and 8 rats in Group Saline. The apparatus was identical to that used in Experiment 5. With the single exception that no auditory CS was presented, half the rats in each group were preexposed in the same manner as was Group Different in Experiment 5, whereas the remainder were preexposed in the same manner as was Group Same. The procedure for conditioning, extinction, and test was the same as that for Experiment 5.

\section{Results}

The mean $( \pm$ SEM $)$ percentage of observations scored as freezing across the conditioning, extinction, and test stages is shown in Figure 6. There was no freezing observed during the context preexposures. Preliminary analyses showed no differences within groups as a consequence of which context they had been preexposed to (same, $n=4$ per group, or different, $n=4$ per group), so data were collapsed across this manipulation for analyses and reporting. Inspection of Figure 6 suggests that s.c. injection of MK-801 prior to extinction training again impaired expression of freezing during extinction training and also impaired extinction learning as indexed by performances on the drug-free test.

During conditioning, the average levels of freezing across the three CS presentations were significantly higher than that of the pre-CS period, $F(1,15)=196.9, p<.05$. A within-subject analysis showed a significant linear increase in freezing during fear acquisition, $F(1,15)=696.9, p<.05$.

During extinction, there were significantly greater levels of pre-CS freezing in Group Saline than in Group MK-801, $F(1$, $14)=31.5, p<.05$. Averaged across groups, there was a significant increase in freezing from pre-CS to first-CS presentation, $F(1,14)=141.2, p<.05$. This increase in freezing interacted significantly with the drug factor, such that it was greater for Group Saline than for Group MK-801, $F(1,14)=106.9, p<.05$. The average level of freezing across the extinction CS presentations was higher for Group Saline than for Group MK-801, $F(1$, $14)=49.8, p<.05$, indicating disruption of freezing by injections of MK-801. Averaged across groups, there was a significant linear decrease in freezing across the extinction session, $F(1,14)=42.8$, $p<.05$. This decrease interacted significantly with the drug factor, $F(1,14)=37.3, p<.05$. These results confirm that extinction training caused significant within-session extinction and that MK801 administration led to disruption of the freezing response.

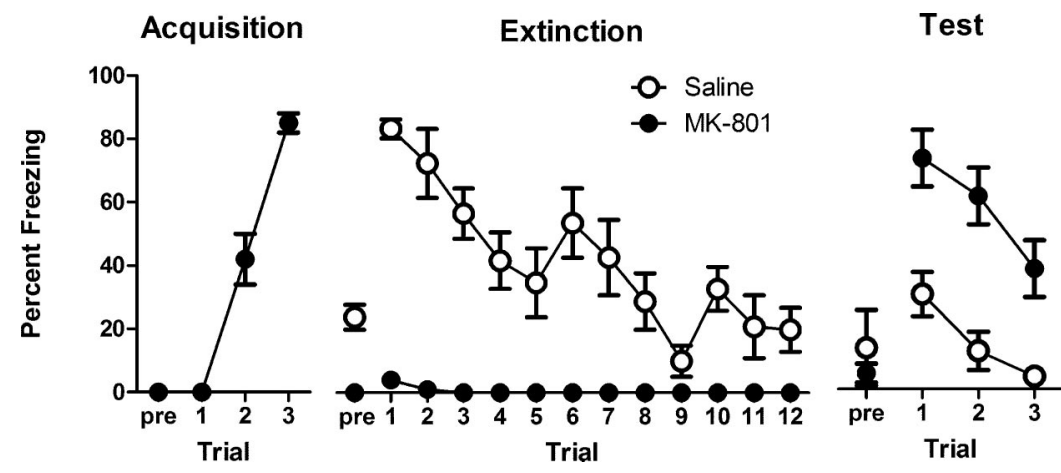

Figure 6. Mean and SEM levels of freezing during Experiment 6. Systemic MK-801 $(0.1 \mathrm{mg} / \mathrm{kg})$ administered prior to initial extinction training impaired long-term extinction learning about a relatively novel conditioned stimulus in groups that had received context preexposures. 
On test, there was no significant difference between Group MK-801 and Group Saline in the level of pre-CS freezing, $F(1$, $14)<1, p>$.05. There was overall significantly more freezing to the CS compared to the pre-CS period $F(1,14)=26.2, p<.05$, and this was greater in Group MK-801 than Saline, $F(1,14)=$ $10.5, p<.05$. There was an overall significant difference between Group MK-801 and Group Saline in freezing across test presentations of the CS, $F(1,14)=20.2, p<.05$. There was a significant reduction in freezing across test trials, $F(1,14)=42.4, p<.05$, but no Group $\times$ Trial interaction, $F(1,14)<1, p>.05$.

These results show that context preexposures did not prevent the impairment of fear extinction learning to an auditory CS by MK801. Taken together with the other results reported here, it appears that familiarity of the target CS is the critical determinant of the effects of MK-801 on fear extinction.

\section{General Discussion}

It is widely accepted that NMDA receptors are critical for the acquisition of conditioned fear and its loss via extinction training. In the case of fear extinction it has been consistently demonstrated that systemic or amygdala infusions of NMDA receptor antagonists impair fear-extinction learning (Myers \& Davis, 2007). Likewise, systemic or intraamygdala infusions of an NMDA receptor partial agonist can facilitate fear-extinction learning. However, recent findings have challenged the necessity of NMDA receptor involvement in fear extinction. For example, fear-extinction learning in infant rats is unaffected by NMDA receptor antagonism (Langton et al., 2007). Moreover, fear reextinction in adult rats can be insensitive to intraamygdala infusions of NMDA receptor antagonists (Laurent et al., 2008) or to systemic administrations of the NMDA receptor partial agonist DCS (Langton \& Richardson, in press). The present experiments showed that familiarity of the $\mathrm{CS}$ is a cause of these differences in involvement of NMDA receptors in fear extinction.

Experiment 1 replicated previous demonstrations that systemic administrations of MK-801 prior to extinction training impaired long-term extinction. Experiment 2 extended previous research by showing that systemic administrations of MK-801 prior to reextinction training did not impair long-term retention of reextinction. These results are representative of the dissociation reported in the literature between sensitivity of initial extinction and reextinction learning to NMDA receptor antagonism and show for the first time that similar results can be obtained with MK-801. The possibility examined here was that familiarity of the CS may be at least one cause for such differences between extinction and reextinction. A CS that is subjected to reextinction training is by definition more familiar than is a CS subjected to initial extinction training, because the reextinguished CS has already been subjected to extinction training. Experiments 3 and 4 manipulated the familiarity of the CS to examine the impact on reextinction and extinction. Experiment 3 showed that MK-801 did significantly impair reextinction learning about a CS that was relatively novel by virtue of having received limited initial extinction training. This effect was modest. However, it is difficult to arrange a set of parameters that allow for significant initial extinction learning without a large number of CS presentations (or long CS durations) that inevitably also increase familiarity of the CS. Demonstrations of the sensitivity of reextinction learning to NMDA receptor antagonism are important because they argue strongly against the possibility that reextinction learning is necessarily qualitatively different to initial extinction learning. For example, as was noted previously, one possible explanation for the apparent dissociation between NMDA receptor contributions to reextinction and extinction is that during reextinction, subjects may simply be able to retrieve the memory acquired during extinction training. That is, reconditioning does not erase the original extinction memory (Rescorla, 2001), and during reextinction training, subjects may retrieve the extinction memory. Such retrieval may be an NMDA receptor-independent process and simply require detection of the nonreinforcement contingency at the start of reextinction. The results of Experiment 3 argue against this possibility because there was evidence for significant extinction during initial extinction training, but reextinction was impaired by MK-801.

Experiments 4 and 5 showed that initial extinction learning can be insensitive to NMDA receptor antagonism. In these experiments the CS was rendered relatively familiar via preexposure. Systemic MK-801 failed to impair initial extinction learning about the relatively familiar CS in Experiment 4. Experiment 5 replicated this effect while at the same time showing that MK-801 did significantly impair initial extinction learning about a relatively novel CS. To the best of our knowledge, this is the first time that initial fear-extinction learning has been shown to be insensitive to NMDA receptor antagonism in adult rats (cf. Langton et al., 2007). It might be argued that preexposure enabled animals to form a memory of the CS being "safe" and that the animal could retrieve this memory during extinction training. In other words, preexposure and extinction share a common memorial feature in addition to their procedural similarity. This interpretation of preexposure effects is contentious and, regardless, is not supported by the observation that a latently inhibited CS is impaired in transformation into an inhibitor of fear (Rescorla, 1971). The data presented here do not support this interpretation. There was no evidence from Experiment 5 that the preexposure regime was sufficient to produce latent inhibition: There were no differences between preexposed and non-pre-exposed groups in fear acquisition or withinsession fear extinction. Rather, the results are consistent with the claim that familiarity of the CS determines the effects of NMDA receptor antagonism on fear-extinction learning.

We tested one possible mechanism of CS familiarity by examining the influence of context change. The rationale was that a CS is familiar or unsurprising by virtue of formation of context-CS associations during initial extinction training (in the case of reextinction) or during preexposure (Wagner, 1981). Such context-CS associations have important implications for learning about and responding to an extinguished CS (Westbrook, Iordanova, McNally, Richardson, \& Harris, 2002). If so, then presentation of that $\mathrm{CS}$ in a second, different context should have reinstated novelty of the CS and also sensitivity to MK-801. It did not reinstate sensitivity to MK-801. A different possibility is that exposures to the $\mathrm{CS}$, during preexposure or initial extinction, simply allowed subjects to learn about the CS itself. This could have been in the form of perceptual learning (for a review, see Hall, 1991). Perceptual learning, which can refer to a learned change in the salience and discriminability of a stimulus, is a well-documented and widespread consequence of stimulus exposure, and it profoundly alters how a subject learns about the CS during subsequent conditioning (Hall, 1991). 
These results are consistent with studies from spatial learning (Bannerman et al., 1995; Saucier \& Cain, 1995; Saucier et al., 1996), Pavlovian contextual fear conditioning (Sanders \& Fanselow, 2003) and one-trial inhibitory avoidance learning (Roesler et al., 1998), showing that the preexposure to the stimuli or actions to be learned about can determine the effects of NMDA receptor antagonism on later learning. In the case of fear extinction, the present results raise the question: What aspect of fearextinction learning do NMDA receptor antagonists normally disrupt? Contemporary accounts suggest that NMDA receptors are the substrate for initiating extinction association or memory formation (Myers \& Davis, 2007; Quirk \& Mueller, 2008). The present results show that extinction association or memory formation can proceed apparently normally in the presence of an NMDA receptor antagonist. One possibility is that extinction learning about a relatively familiar CS is associatively different to extinction learning about a relatively novel CS. For example, perhaps extinction learning about a familiar CS depends on habituationlike processes. A simpler possibility is that NMDA receptor antagonists normally impair extinction learning because they reduce the salience of the events being learned about. Preexposure to the CS protects it from these disruptive effects, possibly because it supports perceptual learning about the CS, allowing it to subsequently enter into associations despite the presence of NMDA receptor antagonists. Regardless of the eventual merit of this account, the important point is that demonstrations such as those provided here and elsewhere (Langton et al., 2007; Langton \& Richardson, in press; Laurent et al., 2008) are not anticipated by contemporary accounts of the role of NMDA receptors in fearextinction learning.

It is worth commenting on the relationship between the effects of MK-801 during extinction and reextinction training and later tests for long-term extinction and reextinction. In each of the experiments reported here, MK-801 disrupted expression of the freezing conditioned response (CR). This disruption occurred independently of the familiarity of the CS, independently of whether the injection preceded extinction or reextinction training, and independently of whether the injection actually disrupted learning. The impairment of extinction learning by MK- 801 has been reported previously under conditions in which the drug did not disrupt freezing (Langton et al., 2007). The dissociation between the sensitivity of initial fear-extinction learning and fearreextinction learning has been reported for other NMDA receptor antagonists (Laurent et al., 2008) and for the facilitatory effects of the NMDA partial agonist DCS (Langton \& Richardson, in press). Thus, there is little reason to suppose that the findings reported here, which confirm that initial extinction learning and reextinction learning are differentially sensitive to NMDA receptor manipulations and identify CS familiarity as a cause of these differences, were due either to the choice of NMDA receptor antagonist or to its motoric effects. Indeed, the data presented here argue strongly against this possibility because across six different experiments there was no evidence that the disruption of freezing by MK-801 was related to its effect on extinction learning. In the experiments reported here MK-801 consistently impaired expression of the fear CR regardless of the presence versus absence of long-term extinction or reextinction. In other words, the amount of freezing observed during extinction training was unrelated to the amount of extinction observed on test among MK-801-treated animals. It is therefore highly unlikely that the effects reported here on extinction and reextinction learning, assessed on the drug-free test, were secondary to effects of the drug on locomotor activity during extinction and reextinction training. Nonetheless, it is worth examining whether similar effects of CS familiarity will be observed with administrations of NMDA receptor antagonists that do not disrupt fear CR expression.

Finally, with the exception of Experiments 5 and 6, all procedures here were carried out in the same context. This raises two issues that might be discussed. First, there was no evidence for differences between groups in pre-CS (i.e., context) freezing on test in these experiments. Laurent et al. (2008) have shown differences in sensitivity of initial extinction and reextinction of context fear conditioning to NMDA receptor antagonism. That there were no such differences in the present experiments is unsurprising: Levels of context-conditioned fear were low throughout tests in these experiments $(<30 \%)$, and the procedures were not designed to provide optimal assessment of contextual fear conditioning. The second issue is the extent to which freezing levels to the CS were contaminated by freezing to the context. With the exception of Experiment 4, levels of freezing to the CS on test were significantly higher than during the pre-CS period. The absence of such differences in Experiment 4 is not of concern because such differences were found in Experiment 5, which replicated the critical differences between groups in CS freezing. Thus, across the experiments reported here, the influence of CS preexposure on the effects of MK-801 was independent of levels of context-elicited freezing.

In conclusion, we studied the role of CS familiarity in influencing the effects of NMDA receptor antagonism on fear-extinction learning. MK-801 consistently impaired fear-extinction learning about a relatively novel CS and consistently failed to impair extinction learning about a relatively familiar CS. This occurred regardless of whether the CS was rendered familiar via initial extinction training or via simple preexposures and regardless of whether a particular fear-extinction episode represented an initial experience or a reexperience. The results show for the first time that CS familiarity is a critical determinant of the effects of MK-801 on fear-extinction learning.

\section{References}

Baker, J. D., \& Azorlosa, J. L. (1996). The NMDA antagonist MK-801 blocks the extinction of Pavlovian fear conditioning. Behavioral Neuroscience, 110, 618-620.

Bannerman, D. M., Good, M. A., Butcher, S. P., Ramsay, M., \& Morris, R. G. (1995). Distinct components of spatial learning revealed by prior training and NMDA receptor blockade. Nature, 378, 182-186.

Cox, J., \& Westbrook, R. F. (1994). The NMDA receptor antagonist MK-801 blocks acquisition and extinction of conditioned hypoalgesic responses in the rat. Quarterly Journal of Experimental Psychology, 47B, 187-210.

Falls, W. A., Miserendino, M. J., \& Davis, M. (1992). Extinction of fear-potentiated startle: Blockade by infusion of an NMDA antagonist into the amygdala. Journal of Neuroscience, 12, 854-863.

Guastella, A. J., Dadds, M. R., Lovibond, P. F., Mitchell, P., \& Richardson, R. (2007). A randomized controlled trial of the effect of D-cycloserine on exposure therapy for spider fear. Journal of Psychiatry Research, 41, $466-471$.

Hall, G. (1991). Perceptual and associative learning. Oxford, England: Oxford University Press. 
Hofmann, S. G., Meuret, A. E., Smits, J. A., Simon, N. M., Pollack, M. H., Eisenmenger, K., et al. (2006). Augmentation of exposure therapy with D-cycloserine for social anxiety disorder. Archives of General Psychiatry, 63, 298-304.

Kim, J. J., DeCola, J. P., Landeira-Fernandez, J., \& Fanselow, M. S. (1991). N-methyl-D-aspartate receptor antagonist APV blocks acquisition but not expression of fear conditioning. Behavioral Neuroscience, 105, 126-133.

Kushner, M. G., Kim, S. W., Donahue, C., Thuras, P., Adson, D., Kotlyar, M., et al. (2007). D-cycloserine augmented exposure therapy for obsessive-compulsive disorder. Biological Psychiatry, 62, 835-838.

Langton, J. M., Kim, J. H., Nicholas, J., \& Richardson, R. (2007). The effect of the NMDA receptor antagonist MK-801 on the acquisition and extinction of learned fear in the developing rat. Learning \& Memory, 14, $665-668$.

Langton, J. M., \& Richardson, R. (in press). D-cycloserine facilitates extinction the first time but not the second time: An examination of the role of NMDA across the course of repeated extinction sessions. Neuropsychopharmacology.

Laurent, V., Marchand, A. R., \& Westbrook, R. F. (2008). The basolateral amygdala is necessary for learning but not relearning extinction of context conditioned fear. Learning \& Memory, 15, 304-314.

Ledgerwood, L., Richardson, R., \& Cranney, J. (2003). Effects of D-cycloserine on extinction of conditioned freezing. Behavioral Neuroscience, 117, 341-349.

Lee, H., \& Kim, J. J. (1998). Amygdalar NMDA receptors are critical for new fear learning in previously fear-conditioned rats. Journal of Neuroscience, $18,8444-8454$.

Miserendino, M. J., Sananes, C. B., Melia, K. R., \& Davis, M. (1990). Blocking of acquisition but not expression of conditioned fearpotentiated startle by NMDA antagonists in the amygdala. Nature, 345 , $716-718$.

Myers, K. M., \& Davis, M. (2007). Mechanisms of fear extinction. Molecular Psychiatry, 12, 120-150.

Quirk, G. J., \& Mueller, D. (2008). Neural mechanisms of extinction learning and retrieval. Neuropsychopharmacology, 33, 56-72.

Rescorla, R. A. (1971). Summation and retardation tests of latent inhibition. Journal of Comparative and Physiological Psychology, 75, 77-81.

Rescorla. R. A. (2001). Retraining of extinguished Pavlovian stimuli.
Journal of Experimental Psychology: Animal Behavior Processes, 27, $115-124$.

Ressler, K. J., Rothbaum, B. O., Tannenbaum, L., Anderson, P., Graap, K., Zimand, E., et al. (2004). Cognitive enhancers as adjuncts to psychotherapy: Use of D-cycloserine in phobic individuals to facilitate extinction of fear. Archive of General Psychiatry, 61, 1136-1144.

Roesler, R., Vianna, M., Sant'Anna, M. K., Kuyven, C. R., Kruel, A. V., Quevedo, J., et al. (1998). Intrahippocampal infusion of the NMDA receptor antagonist AP5 impairs retention of an inhibitory avoidance task: Protection from impairment by pretraining or preexposure to the task apparatus. Neurobiology of Learning and Memory, 69, 87-91.

Sanders, M. J., \& Fanselow, M. S. (2003). Pre-training prevents context fear conditioning deficits produced by hippocampal NMDA receptor blockade. Neurobiology of Learning and Memory, 80, 123-129.

Saucier, D., \& Cain, D. P. (1995). Spatial learning without NMDA receptor-dependent long-term potentiation. Nature, 378, 186-189.

Saucier, D., Hargreaves, E. L., Boon, F., Vanderwolf, C. H., \& Cain, D. P. (1996). Detailed behavioral analysis of water maze acquisition under systemic NMDA or muscarinic antagonism: Nonspatial pretraining eliminates spatial learning deficits. Behavioral Neuroscience, 110, 103116

Sotres-Bayon, F., Bush, D. E., \& LeDoux, J. E. (2007). Acquisition of fear extinction requires activation of NR2B-containing NMDA receptors in the lateral amygdala. Neuropsychopharmacology, 32, 1929-1940.

Wagner, A. R. (1981). SOP: A model of automatic memory processing in animal behavior. In N. E. Spear \& R. R. Miller (Eds.), Information processing in animals: Memory mechanisms (pp. 5-47). Hillsdale, NJ: Erlbaum.

Walker, D. L., Ressler, K. J., Lu, K. T., \& Davis, M. (2002). Facilitation of conditioned fear extinction by systemic administration or intraamygdala infusions of D-cycloserine as assessed with fear-potentiated startle in rats. Journal of Neuroscience, 22, 2343-2351.

Westbrook, R. F., Iordanova, M., McNally, G., Richardson, R., \& Harris, J. A. (2002). Reinstatement of fear to an extinguished conditioned stimulus: Two roles for context. Journal of Experimental Psychology: Animal Behavior Processes, 28, 97-110.

Received August 22, 2008 Revision received November 3, 2008 Accepted November 24, 2008 